\title{
Seroepidemiological evidence of severe fever with thrombocytopenia syndrome virus infections in wild boars in Nagasaki, Japan
}

\author{
Daisuke Hayasaka ${ }^{1,2^{*}}$, Yu Fuxun ${ }^{1}$, Akira Yoshikawa ${ }^{1,3}$, Guillermo Posadas-Herrera ${ }^{1,4}$, Satoshi Shimada ${ }^{1,2}$, \\ Mya Myat Ngwe Tun', Masanobu Agoh ${ }^{1,3}$ and Kouichi Morita ${ }^{1,2}$
}

\begin{abstract}
Severe fever with thrombocytopenia syndrome (SFTS) is an emerging disease in East Asia. It is thought that the SFTS virus (SFTSV) circulates between ticks and animals in nature and that the virus is transmitted to humans by tick bites. SFTS is endemic to Nagasaki in western Japan; however, epidemiological information regarding SFTSV in Nagasaki is not known. In this study, we performed SFTSV IgG ELISAs and neutralization antibody assays for a seroepidemiological survey using samples from wild boars captured in six areas of Nagasaki. SFTSV seropositive animals were found in three areas. Our findings provide epidemiological information on the distribution of SFTSV in Nagasaki.
\end{abstract}

\section{Introduction}

Severe fever with thrombocytopenia syndrome (SFTS) is an emerging disease that was first reported in China and has been identified in South Korea and Japan [1-4]. The causative agent, SFTS virus (SFTSV), belongs to the genus Phlebovirus in the family Bunyaviridae [4]. Humans appear to be infected by the bite of an infected tick, such as Haemaphysalis longicornis [5]. Seroepidemiological surveys have demonstrated that anti-SFTSV antibodies have been identified in domestic and wild animals, including sheep, cattle, and dog, in endemic area of SFTS [6-8], indicating that SFTSV circulates between ticks and animals in nature.

The clinical symptoms of SFTS include fever, enteritis, thrombocytopenia, and leukopenia, with fatality rates of up to $30 \%[2,4,9,10]$. No specific treatment or vaccines for SFTS are currently available. Thus, an epidemiological survey that provides distribution of SFTSV in ticks and animals will be of help for the prevention of the disease in endemic areas.

\footnotetext{
* Correspondence: hayasaka@nagasaki-u.ac.jp

${ }^{1}$ Department of Virology, Institute of Tropical Medicine (NEKKEN), Nagasaki University, 1-12-4 Sakamoto, Nagasaki 852-8523, Japan

${ }^{2}$ Leading Graduate School Program, Nagasaki University, 1-12-4 Sakamoto,

Nagasaki 852-8523, Japan

Full list of author information is available at the end of the article
}

In Japan, more than 140 cases of SFTS have been identified since 2005 http://kanpoken.pref.yamaguchi.lg.jp/ jyoho/page9/sfts_1.php. In Nagasaki located on the Japanese island of Kyushu, seven cases were identified by 2014 [2]. We previously reported that neither virus isolation nor viral gene detection was confirmed in tick pools that included more than 2000 ticks collected in Nagasaki [11]. This indicates that the epidemiological survey of SFTSV in ticks may not provide enough information on the distribution of SFTSV in the region. Alternatively, seroepidemiological surveys in animals can provide this information.

In this study, we attempted to identify anti-SFTSV seropositive animals by using serum samples of wild boars that were captured in Nagasaki, and we examined the infectious rates and localities of these animals.

\section{Methods \\ Virus and cells \\ The YG-1 strain of SFTSV was kindly provided by Ken Maeda, Yamaguchi University. The NagH2013-1 strain of SFTSV was isolated from an SFTS patient in Nagasaki in 2013. Vero E6 cells were maintained in Eagle's minimal essential medium (EMEM; Nissui Pharmaceutical Co.) containing $10 \%$ fetal bovine serum (FBS). Stock SFTSV}


was prepared from the cell culture medium of Vero E6 cells in EMEM containing 2 \% FBS. Virus titers were determined by a focus forming assay [12]. Briefly, confluent Vero E6 cells were inoculated with serially diluted culture supernatants of SFTSV and incubated in $2 \%$ FBS EMEM containing $1 \%$ methyl cellulose 4000 (Wako Pure Chemical Industries, Ltd.) for 5 days. Viral foci were detected by using SFTSV antiserum (source: recovered SFTS human case), peroxidase-conjugated antihuman IgG (American Qualex), and the DAB substrate (Wako Pure Chemical Industries, Ltd.). Virus titers were expressed as focusforming units (ffu) per milliliter. The experiment using human serum was performed with the approval of the ethics committee of the Institute of Tropical Medicine, Nagasaki University (approval number: 140829129). All experiments using live SFTSV were performed in a biosafety level 3 laboratory at Nagasaki University according to standard BSL3 guidelines.

\section{Serum samples of wild boar}

A total of 190 serum samples were collected from wild boars that were captured in six areas of the Nagasaki prefecture (Fig. 1) from 2006 to 2012 for wild boar control conducted by Nagasaki prefecture. Samples were from juvenile (184 samples) and adult (6 samples) animals. The sera were inactivated at $56{ }^{\circ} \mathrm{C}$ for $30 \mathrm{~min}$.

\section{Indirect IgG ELISA using recombinant SFTSV-N protein}

Recombinant SFTSV-N protein was expressed and purified as previously described [13]. Recombinant Rift Valley fever virus (RVFV)-N protein was expressed and purified using the same procedure. The odd-numbered wells of rows A-G of 96-well Nunc immunoplates (Thermo Scientific, Denmark) were coated with $100 \mu \mathrm{l}(50 \mathrm{ng} /$ well $)$ of recombinant SFTSV-N protein (positive antigen), and the evennumbered wells of the same rows of the plates were coated with $100 \mu \mathrm{l}(50 \mathrm{ng} /$ well) of RVFV-N protein (negative antigen) in PBS at $\mathrm{pH}$ 7.2. The plates were left at $4{ }^{\circ} \mathrm{C}$ overnight. After blocking the wells with $5 \%$ nonfat milk (Difco, Detroit, USA) in PBS containing $0.1 \%$ Tween 20 (PBS-T) for $1 \mathrm{~h}$ at $37{ }^{\circ} \mathrm{C}$, the plates were washed three times with PBS-T. A $100-\mu \mathrm{l}$ volume of wild boar serum diluted 1:100 with $5 \%$ nonfat milk in PBS-T was then added to all wells previously added with either the positive or the negative antigen. Plates were incubated for $1 \mathrm{~h}$ at $37^{\circ} \mathrm{C}$ after which they were washed as above. Then, $100 \mu \mathrm{l}$ of 1:10,000 diluted horseradish peroxidase-conjugated goat anti-pig IgG (Bethyl Laboratories Inc., USA) was added, and incubation was done for $1 \mathrm{~h}$ at $37{ }^{\circ} \mathrm{C}$. After washing the plates, $100 \mu \mathrm{l}$ of $\mathrm{H}_{2} \mathrm{O}_{2}$-ABTS

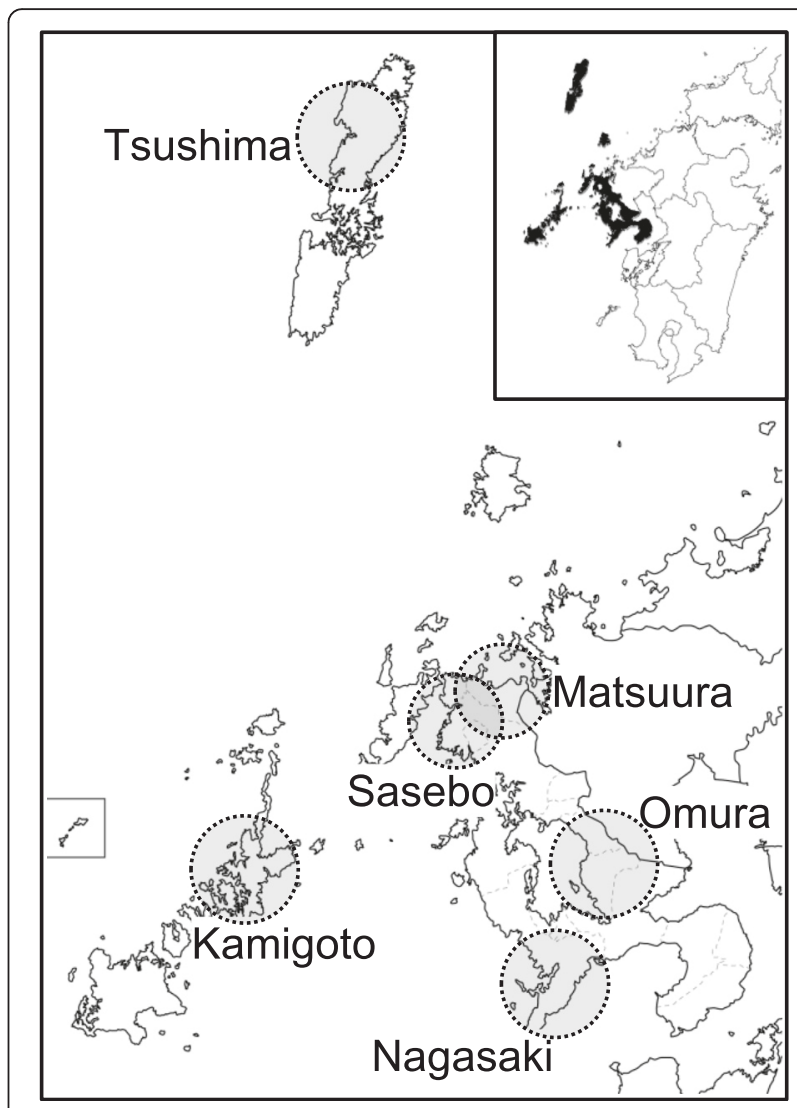

Fig. 1 Map of the Nagasaki prefecture in the Kyushu islands, Japan. Circles indicate the areas where wild boars were captured. Inset map: Kyushu islands with the darkened areas indicating theNagasaki prefecture

substrate (Kirkegaard \& Perry, Gaithersburg, MD) was added to all the wells. After incubation at $37{ }^{\circ} \mathrm{C}$ for $30 \mathrm{~min}$, optical density (OD) values at $410 \mathrm{~nm}$ were measured. Each serum sample was tested in duplicate. The adjusted OD was calculated by subtracting the OD of the well with the negative antigen from that with the positive antigen. The OD cutoff value was calculated as the mean of the adjusted OD of the negative control sera plus three times the standard deviations, generally an OD of $\leq 0.2$ at a 1:100 sample dilution. Five negative control samples were randomly selected from negative sera determined by focus reduction neutralization test as described below. Serum samples were considered to be ELISA positive if the adjusted OD value was greater than or equal to the assay cutoff value of 0.2 .

\section{The FRNT}

Neutralizing antibody titers based on $80 \%$ focus reduction neutralization test (FRNT) were determined for serum samples found positive by IgG ELISA. Each serum sample at different serial twofold dilutions (1:20 to 1:1280) was mixed with SFTSV (50 ffu) and 
incubated at $37{ }^{\circ} \mathrm{C}$ for $1 \mathrm{~h}$. Each mixture of SFTSV and diluted serum sample was inoculated with duplicate in Vero E6 cells which were then incubated in 2 \% FCS EMEM containing $1 \%$ methyl cellulose 4000 for 5 days. Detection of foci of infected cells was performed as described above in the focus forming assay protocol. The neutralizing titer was determined as the reciprocal of the highest serum dilution that reduced viral foci counts by $80 \%$. Samples showing more than a titer of 1:20 were considered to be FRNT positive. However, we were able to observe that wild animal serum exhibited nonspecific inhibitory activity against virus infections not due to specific neutralizing antibody and few samples just below 0.2 of OD value showed low FRNT titers. Thus, in this study, we considered that samples showing both $>0.2$ OD value (ELISA) and $>1: 20$ FRNT titer were seropositive for SFTSV.

\section{Results and discussion}

The serum samples of wild boars were first examined by indirect IgG ELISA for the detection of past infection with SFTSV. Wild boars with IgG seropositive samples were detected in four out of the six study areas in Nagasaki prefecture (Fig. 2). The IgG seropositive rates were $51,25,0,19,0$, and $1.4 \%$ in Nagasaki, Omura, Sasebo, Matsuura, Tsushima, and Kamigoto, respectively (Fig. 2).

We next performed an FRNT assay to confirm the positive results obtained from the IgG ELISA. All of the samples except one (K23-15) showed FRNT antibody titers of more than 1:20 (Table 1). Considering together the results of indirect IgG ELISA and FRNT, the number of SFTSV seropositive wild boars was 27 out of 53 (51\%), 1 out of 4 ( $25 \%)$, and 7 out of $37(19 \%)$ in the areas of Nagasaki, Omura, and Matsuura, respectively. On the other hand, no seropositive wild boars were identified in Sasebo, Tsushima, and Kamigoto. There were no significant differences of positive rates between juvenile $(16.7 \%)$ and adult animals (19.0\%). Also, due to the limited number of samples, there were no significant differences in the positive rates in each area during 2006 to 2012 (data not shown). These results suggest that in the areas where SFTSV seropositive animals
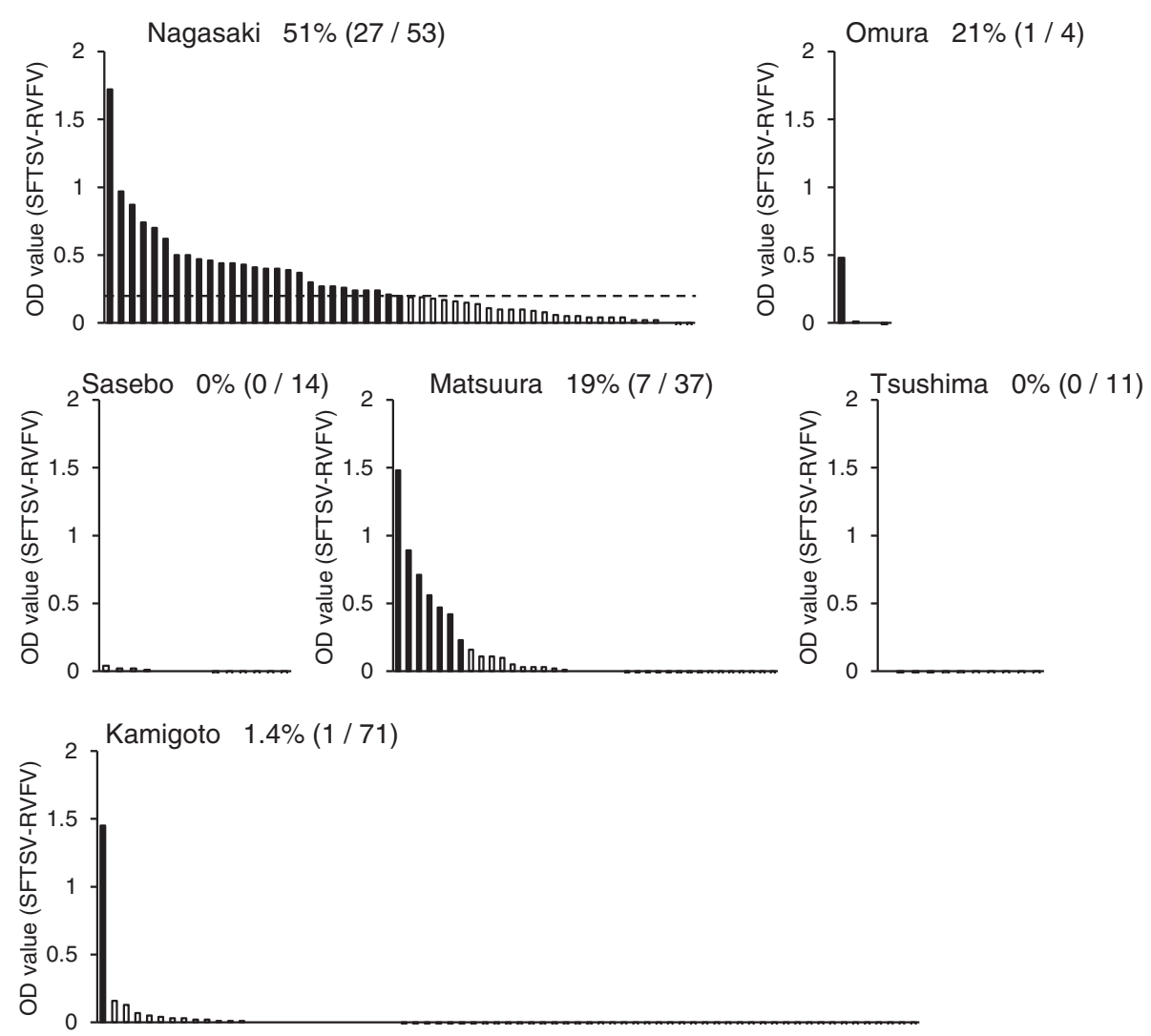

Fig. 2 OD values after IgG ELISA of the serum samples of wild boars caught in the six areas of the Nagasaki prefecture. The $y$-axis indicates the OD values calculated by subtracting each of the OD value of the sample with the negative antigen (RVFV antigen) from the OD value of the same sample with the positive antigen (SFTSV antigen). OD cutoff value was 0.2 
Table 1 FRNT antibody titers of wild boars

\begin{tabular}{|c|c|c|}
\hline Sample no. & $\mathrm{ELISA}^{a}$ & FRNT \\
\hline \multicolumn{3}{|l|}{ Nagasaki } \\
\hline $\mathrm{N} 21-22$ & 1.72 & $1: 80$ \\
\hline $\mathrm{N} 21-20$ & 0.97 & $1: 160$ \\
\hline N21-15 & 0.87 & $1: 160$ \\
\hline N21-32 & 0.74 & $1: 640$ \\
\hline N21-68 & 0.70 & $1: 80$ \\
\hline N19-42 & 0.62 & $1: 160$ \\
\hline N21-8 & 0.50 & $1: 320$ \\
\hline N21-28 & 0.50 & $1: 320$ \\
\hline N21-53 & 0.47 & $1: 320$ \\
\hline N19-38 & 0.46 & $1: 160$ \\
\hline N21-17 & 0.44 & $1: 40$ \\
\hline N21-45 & 0.44 & $1: 320$ \\
\hline N19-30 & 0.43 & $1: 320$ \\
\hline N21-33 & 0.41 & $1: 80$ \\
\hline N21-6 & 0.40 & $1: 80$ \\
\hline N21-31 & 0.40 & $1: 160$ \\
\hline N21-34 & 0.39 & $1: 640$ \\
\hline N21-60 & 0.37 & $1: 320$ \\
\hline N19-26 & 0.30 & $1: 160$ \\
\hline N21-13 & 0.27 & $1: 160$ \\
\hline N21-24 & 0.27 & $1: 160$ \\
\hline N19-23 & 0.26 & $1: 320$ \\
\hline N20-3 & 0.24 & $1: 160$ \\
\hline N21-25 & 0.24 & $1: 160$ \\
\hline N21-65 & 0.24 & $1: 160$ \\
\hline N21-16 & 0.21 & $1: 320$ \\
\hline N19-39 & 0.20 & $1: 160$ \\
\hline \multicolumn{3}{|l|}{ Matsuura } \\
\hline M21-4 & 1.48 & $1: 160$ \\
\hline M23-1 & 0.89 & $1: 640$ \\
\hline M24-1 & 0.71 & $1: 20$ \\
\hline M21-17 & 0.56 & $1: 640$ \\
\hline M21-11 & 0.47 & $1: 160$ \\
\hline M21-9 & 0.42 & $1: 20$ \\
\hline M22-11 & 0.23 & $1: 20$ \\
\hline \multicolumn{3}{|l|}{ Omura } \\
\hline O23-1 & 0.48 & $1: 160$ \\
\hline \multicolumn{3}{|l|}{ Kamigoto } \\
\hline K23-15 & 1.45 & $<1: 20$ \\
\hline
\end{tabular}

${ }^{a} \mathrm{OD}$ value (SFTSV-RVFV) were found, SFTSV may be distributed in ticks and that wild boars may be frequently exposed to SFTSV.

Although a sample of K23-15 (Kamigoto sample) showed high ELISA OD value (1.45), the FRNT antibody titer was less than 1:20 (Table 1). This raises the possibility that this animal might be infected with another virus closely related to SFTSV. Recently, new pathogenic tick-borne viruses have been reported globally, and some tick-borne viruses belonging to the genus Phlebovirus of the family Bunyaviridae have been reported $[14,15]$. The earliest reported case of SFTS worldwide was from a patient in Nagasaki who was infected in 2005, suggesting that SFTSV has likely existed in this country for a long time. This raises the possibility that unknown viruses are also distributed in this area. Indeed, we isolated a new virus belonging to the genus Nairovirus of the family Bunyaviridae from ticks in Kamigoto (Shimada et al., unpublished results). Thus, further investigation of tick-borne viruses, including SFTSV and unidentified viruses in this area, will provide useful information for the development of pre-emptive countermeasures against potentially pathogenic tick-borne diseases.

In this study, we demonstrated that wild boars are likely to be useful sentinels for identifying the distributions of SFTSV. Other groups have identified SFTSV seropositive areas in Japan using animal samples from deer and dogs http://www.nih.go.jp/niid/ja/id/2244disease-based/sa/sfts/idsc/iasr-in/4491-pr4094.html.

\section{Competing interests}

The authors declare that they have no competing interests.

\section{Authors' contributions}

$\mathrm{DH}$ and KM designed the study; DH, YF, AY, PHG, SS, and NTMM performed the experiments; DH, MA, and KM analyzed the data; and DH and YF wrote the paper. All authors read and approved the final manuscript.

\section{Acknowledgements}

We thank Ken Maeda (Joint Faculty of Veterinary Medicine, Yamaguchi University) for providing the SFTSV YG-1 and Koichi Izumikawa (Department of Infectious Diseases, Graduate School of Biomedical Sciences, Course of Emerging Infectious Diseases, Nagasaki University) for providing the antiserum against SFTS. This work was supported financially by KAKENHI [Grant-in-Aid for Scientific Research (B) (25304045)] from the Japan Society for the Promotion of Science and also by a Health and Labor Sciences Research Grant on Emerging and Re-emerging Infectious Diseases from the Japanese Ministry of Health, Labor and Welfare, Health and Labor Sciences Research Grants, AMED, and the Japan Initiative for Global Research Network on Infectious Diseases.

\section{Author details}

${ }^{1}$ Department of Virology, Institute of Tropical Medicine (NEKKEN), Nagasaki University, 1-12-4 Sakamoto, Nagasaki 852-8523, Japan. ${ }^{2}$ Leading Graduate School Program, Nagasaki University, 1-12-4 Sakamoto, Nagasaki 852-8523, Japan. ${ }^{3}$ Nagasaki Prefectural Institute for Environmental Research and Public Health, Omura, Nagasaki 856-0026, Japan. ${ }^{4}$ Present address: National Institute of Infectious Diseases, 1-23-1 Toyama, Shinjuku-ku, Tokyo 162-8640, Japan.

Received: 26 November 2015 Accepted: 2 March 2016

Published online: 03 April 2016 


\section{References}

1. Kim KH, Yi J, Kim G, et al. Severe fever with thrombocytopenia syndrome, South Korea, 2012. Emerg Infect Dis. 2013;19:1892-4.

2. Takahashi T, Maeda K, Suzuki T, et al. The first identification and retrospective study of severe fever with thrombocytopenia syndrome in Japan. J Infect Dis. 2014;209:816-27.

3. Xu B, Liu L, Huang $X$, et al. Metagenomic analysis of fever, thrombocytopenia and leukopenia syndrome (FTLS) in Henan Province, China: discovery of a new bunyavirus. PLoS Pathog. 2011;7:e1002369.

4. Yu XJ, Liang MF, Zhang SY, et al. Fever with thrombocytopenia associated with a novel bunyavirus in China. N Engl J Med. 2011;364:1523-32.

5. Zhang YZ, Zhou DJ, Qin XC, et al. The ecology, genetic diversity, and phylogeny of Huaiyangshan virus in China. J Virol. 2012;86:2864-8.

6. Ding $\mathrm{S}$, Yin $\mathrm{H}, \mathrm{Xu} \mathrm{X}$, et al. A cross-sectional survey of severe fever with thrombocytopenia syndrome virus infection of domestic animals in Laizhou City, Shandong Province, China. Jpn J Infect Dis. 2014;67:1-4.

7. Li Z, Hu J, Bao C, et al. Seroprevalence of antibodies against SFTS virus infection in farmers and animals, Jiangsu, China. J Clin Virol. 2014;60:185-9.

8. Niu G, Li J, Liang M, et al. Severe fever with thrombocytopenia syndrome virus among domesticated animals, China. Emerg Infect Dis. 2013;19:756-63.

9. Liu Q, He B, Huang SY, Wei F, Zhu XQ. Severe fever with thrombocytopenia syndrome, an emerging tick-borne zoonosis. Lancet Infect Dis. 2014;14:763-72.

10. Liu S, Chai C, Wang C, et al. Systematic review of severe fever with thrombocytopenia syndrome: virology, epidemiology, and clinical characteristics. Rev Med Virol. 2014;24:90-102.

11. Hayasaka D, Shimada S, Aoki K, et al. Epidemiological survey of severe fever with thrombocytopenia syndrome virus in ticks in Nagasaki, Japan. Trop Med Health. 2015;43:159-64.

12. Shimada S, Posadas-Herrera G, Aoki K, Morita K, Hayasaka D. Therapeutic effect of post-exposure treatment with antiserum on severe fever with thrombocytopenia syndrome (SFTS) in a mouse model of SFTS virus infection. Virology. 2015;482:19-27.

13. Yu F, Du Y, Huang $X$, et al. Application of recombinant severe fever with thrombocytopenia syndrome virus nucleocapsid protein for the detection of SFTSV-specific human IgG and IgM antibodies by indirect ELISA. Virol J. 2015;12:117.

14. Matsuno K, Weisend C, Kajihara M, et al. Comprehensive molecular detection of tick-borne phleboviruses leads to the retrospective identification of taxonomically unassigned bunyaviruses and the discovery of a novel member of the genus phlebovirus. J Virol. 2015;89:594-604.

15. Matsuno K, Weisend C, Travassos da Rosa AP, et al. Characterization of the Bhanja serogroup viruses (Bunyaviridae): a novel species of the genus Phlebovirus and its relationship with other emerging tick-borne phleboviruses. J Virol. 2013:87:3719-28.

\section{Submit your next manuscript to BioMed Central and we will help you at every step:}

- We accept pre-submission inquiries

- Our selector tool helps you to find the most relevant journal

- We provide round the clock customer support

- Convenient online submission

- Thorough peer review

- Inclusion in PubMed and all major indexing services

- Maximum visibility for your research

Submit your manuscript at www.biomedcentral.com/submit

CBiomed Central 